\title{
ON THE TOPOLOGICAL PRODUCT OF PARACOMPACT SPACES
}

\author{
R. H. SORGENFREY
}

A space $S$ is defined by Jean Dieudonné ${ }^{1}$ to be paracompact provided every covering of $S$ by open sets has a neighborhood-finite refinement ${ }^{2}$ which covers it. Dieudonné proves that every metric separable space is paracompact, that every paracompact Hausdorff space is normal, and that if $S_{1}$ is compact and $S_{2}$ is paracompact, then $S_{1} \times S_{2}$ is paracompact. He leaves open the question as to whether the topological product of two paracompact spaces is paracompact. It is the purpose of this note to answer this question in the negative. The example given provides also a simple instance of a normal Hausdorff space $S$ such that $S \times S$ is not normal.

The space $S$. The points of the space $S$ are the non-negative real numbers, and a neighborhood of a point is any semi-open interval of the form $a \leqq x<b$ which contains it. It is easily seen that $S$ is a regular Hausdorff space which is separable but not perfectly separable.

To show that $S$ is paracompact, let $\alpha$ be any covering of $S$ by open sets. It is evident that there is a refinement $\beta$ of $\alpha$ whose elements are neighborhoods. Let $E$ be the set of all points $e$ of $S$ which satisfy the relation $a<e<b$ for no neighborhood $a \leqq x<b$ of $\beta$. The set $E$ is closed with respect to the natural topology of the real numbers. (For if $z$ is a point in the complement of $E$, there is a neighborhood $a \leqq x<b$ of $\beta$ such that $a<z<b$, and since the open interval $a<x<b$ contains no point of $E$, it follows that $z$ is not a limit point of $E$.) The complement of $E$, therefore, is the sum of a countable number of disjoint open intervals $I_{1}, I_{2}, \ldots$. For each $n$ the left end point of $I_{n}$ is a point of $E$, and each point of $E$ is the left end point of some interval $I_{n}$. Hence if $I_{n}$ is the interval $e_{n}<x<f_{n}$, it follows that $E=\sum e_{n}$ and hence that $S=\sum\left(I_{n}+e_{n}\right)$. For each $n$ there exists a neighborhood $e_{n} \leqq x<q_{n}$ of $\beta$; denote it by $D_{n}$. Let $\gamma_{n}$ be the collection of all open intervals $a<x<b$ such that $a \leqq x<b$ belongs to $\beta$ and is a subset of $e_{n} \leqq x<f_{n}$. Then $\gamma_{n}$ covers $q_{n} \leqq x<f_{n}$. Since under the natural topology of the real

Presented to the Society, November 30, 1946; received by the editors January 7, 1947.

1 Une generalisation des espaces compacts, J. Math. Pures Appl. (9) vol. 23 (1944) pp. $65-76$.

2 The covering $\omega$ is a refinement of a covering $\alpha$ if every element of $\omega$ is a subset of some element of $\alpha ; \omega$ is neighborhood-finite if every point of the space has a neighborhood which intersects only a finite number of elements of $\omega$. 
numbers $q_{n} \leqq x<f_{n}$ is paracompact, it follows that there is a neighborhood-finite refinement $\delta_{n}$ of $\gamma_{n}$ which covers it. Let $\epsilon_{n}$ be the collection of intersections of elements of $\delta_{n}$ with $q_{n} \leqq x<f_{n}$. The elements of $\epsilon_{n}$ are open sets in the topology of $S$, and each of them is a subset of an element of $\alpha$. Hence if $\eta_{n}$ is the collection obtained by adding to $\epsilon_{n}$ the open set $D_{n}, \eta_{n}$ is a neighborhood-finite covering of $I_{n}+e_{n}$ whose elements are subsets of elements of $\alpha$; therefore $\eta=\sum \eta_{n}$ is a neighborhood-finite refinement of $\alpha$ which covers $S$.

Hence $S$ is paracompact and therefore normal.

The space $S \times S$. The points of $S \times S$ are, of course, sensed pairs $(x, y)$ of non-negative real numbers, and neighborhoods are semi-open rectangles of the form $a \leqq x<b, c \leqq y<d$.

To show that $S \times S$ is not paracompact it will be sufficient to show that it is not normal. To this end let $H$ be the set of all points $(x, y)$ of $S \times S$ such that $x+y=1$ and $\left[(x-1)^{2}+y^{2}\right]^{1 / 2}$ is rational, and let $K$ be the set of all points $(x, y)$ of $S \times S$ such that $x+y=1$ and $\left[(x-1)^{2}+y^{2}\right]^{1 / 2}$ is irrational. It is readily seen that $H$ and $K$ are disjoint closed sets in $S \times S$. Let $V$ be any open set containing $K$. For each point $\left(k, k^{\prime}\right)$ of $K$ there is a neighborhood $N\left(k, k^{\prime}\right)$ of the form $k \leqq x<k+d, k^{\prime} \leqq y<k^{\prime}+d$ which lies in $V$. For each positive integer $n$ let $K_{n}$ be the set of all points $\left(k, k^{\prime}\right)$ of $K$ such that the diameter of $N\left(k, k^{\prime}\right)$ is greater than $1 / n$. Then $K=\sum K_{n}$, and since $K$ is of the second category with respect to the natural topology of the line interval $x+y=1$, it follows that there exists an integer $m$ such that $K_{m}$ is dense in an interval $(s, 1-s),(t, 1-t)$ of $x+y=1$. It follows easily that every point of the rectangle $R$ bounded by $x+y=1$, $x+y=1+1 / m, x-y=2 s-1, x-y=2 t-1$ belongs to some $N\left(k, k^{\prime}\right)$. Hence $R$ is a subset of $V$. But every point of $H$ in the interval $(s, 1-s),(t, 1-t)$ of $x+y=1$ is a limit point of $R$ and hence of $V$. It follows that $H$ is intersected by the closure of every open set containing $K$.

Hence $S \times S$ is not normal and therefore not paracompact.

University of California at los Angeles 